\title{
Food security status among grade 5 students in Nova Scotia, Canada and its association with health outcomes
}

\author{
Sara FL Kirk 1,2,*, Stefan Kuhle ${ }^{3}$, Jessie-Lee D Mclsaac ${ }^{1,4}$, Patty L Williams ${ }^{5}$, \\ Melissa Rossiter ${ }^{5}$, Arto Ohinmaa ${ }^{4}$ and Paul J Veugelers ${ }^{4}$ \\ ${ }^{1}$ School of Health and Human Performance, Dalhousie University, PO Box 15000, Halifax, NS B3H 4R2, Canada: \\ ${ }^{2}$ MRC Human Nutrition Research, Elsie Widdowson Laboratory, Cambridge, UK: ${ }^{3}$ Perinatal Epidemiology Research \\ Unit, Dalhousie University, Halifax, NS, Canada: ${ }^{4}$ School of Public Health, University of Alberta, Edmonton, AB, \\ Canada: ${ }^{5}$ Department of Nutrition and Dietetics, Mount Saint Vincent University, Halifax, NS, Canada
}

Submitted 6 November 2013: Final revision received 6 May 2014: Accepted 11 June 2014: First published online 30 July 2014

\begin{abstract}
Objective: Food security (FS) exists when all people, at all times, have physical and economic access to sufficient, safe and nutritious food to meet their needs. The present research sought to determine whether students from households experiencing moderate or severe food insecurity (FI) had poorer diet quality, higher body weights and poorer psychosocial outcomes than students from households classed as having high FS or marginal FI status.

Design: Population-based survey conducted in schools. Multiple regression analysis was used to explore associations between FS status (high FS; marginal, moderate, severe FI), dietary behaviours and intake, and health-related outcomes (body weight, quality of life, mood, peer relationships, externalizing problems). Setting: Nova Scotia, Canada.

Subjects: Grade 5 students ( $n$ 5853), aged 10-11 years, with complete information on FS status and student outcomes.

Results: In this sample, rates of household FS were $73.5 \%$ (high FS), $8.3 \%$ (marginal FI) $10 \cdot 2 \%$ (moderate FI) and $7 \cdot 1 \%$ (severe FI status). Students living in households experiencing moderate or severe FI had poorer diet quality, higher BMI and poorer psychosocial outcomes than students classed as having high FS or marginal FI. Conclusions: These findings provide important evidence for policy makers on the prevalence of FI among families in Nova Scotia with grade 5 children and its relationship with childhood nutrition, psychosocial and quality of life factors, and weight status.
\end{abstract}



In 1974, the UN stated that 'every man, woman and child has the inalienable right to be free from hunger and malnutrition in order to develop fully and maintain their physical and mental faculties, ${ }^{,(1)}$. This basic human right to food, also termed food security (FS), exists when 'all people, at all times, have physical and economic access to sufficient, safe and nutritious food to meet their dietary needs and food preferences for an active and healthy life ${ }^{,(2)}$. Households that are food secure have no problems, or anxiety about, consistently accessing adequate and nutritious food. Food insecurity (FI) lies at the other end of the spectrum. At an individual and household level, FI is understood to be a dynamic, managed process, which can range in severity from anxiety and uncertainty regarding food supplies, to compromises in the quantity and quality of food intakes and damaging self-worth when there is a need to acquire foods in socially unacceptable ways, such as through food banks ${ }^{(3)}$. Although FI has been monitored in Canada since the mid-1990s, it has only recently incorporated a validated measure, the Household Food Security Survey Module (HFSSM), that enables comparison of trends over time ${ }^{(4,5)}$. FI can be further subdivided into marginal, moderate or severe $\mathrm{FI}^{(6)}$. Using this measure, FI rates are increasing in Canada, with important regional variations $^{(4)}$. For example, in Nova Scotia, FI affects about $23 \%$ of residents, with one-third of these reporting severe FI, rates that are consistently higher than most other provinces and territories in Canada ${ }^{(7)}$. Research has also found significantly higher prevalence rates among homes with children under the age of 18 years and with three or more children, lone-parent and in particular femaleled households, households receiving social assistance, households who do not own their own home and aboriginal groups ${ }^{(8-10)}$.

FI is directly related to income, and with household expenses such as rent and utilities often taking priority 
over food, this can lead to a range of strategies that can impact nutritional status, such as meal skipping, food coupon use and consumption of less expensive food items $^{(11,12)}$. It is therefore not surprising that research has found that households comprising adults and adolescents with low levels of income and those reliant on social assistance are less likely to purchase fruits, vegetables and milk products or to consume the recommended daily servings of fruits and vegetables ${ }^{(13,14)}$ as well as purchase milk products ${ }^{(15)}$. This translates into low consumption of fruits, vegetables and milk, high amounts of energy-dense food and a higher risk of inadequate nutrient intakes, a pattern that has been shown to contribute to poor health outcomes in adults and adolescents ${ }^{(8)}$. Research has also identified the reportedly 'paradoxical' finding that persons from lower socio-economic backgrounds with fewer resources to purchase food are also more likely to be overweight or obese ${ }^{(16-18)}$, particularly if female ${ }^{(19)}$. For example, household FI is associated with overweight and obesity among mothers, but not child-free women or men ${ }^{(20)}$. A key explanation for this has been suggested as an expression of gendered expectations on women, who themselves may sacrifice their own needs for food to provide for their children $^{(20)}$. However, much of the existing research has been conducted on adults and adolescents, and remains inconsistent, with limited research on how FI influences health outcomes in children, and what evidence is available is mixed $^{(21)}$.

Recently, a conceptual framework was proposed to describe the relationship between poverty, FI and obesity ${ }^{(22)}$. This framework portrays a cycle of mutual influence among the mechanisms (physiological, behavioural and psychosocial-cultural) that translate the environmental and contextual influences of poverty and FI into health outcomes, including obesity ${ }^{(22)}$. Applying a conceptual framework to guide research provides an evidence-based model to understand how FI influences health outcomes. Further conceptual refinement is needed to elucidate the exact relationship between FI, obesity and health outcomes ${ }^{(22)}$, particularly in children, who remain a population for whom there are significant gaps in our knowledge. Therefore, the aim of the present paper was to explore the relationship between FS, diet quality, weight status and psychosocial outcomes, as outlined in this conceptual framework, in grade 5 children participating in a population-based survey in Nova Scotia, Canada. We hypothesized that students from households experiencing moderate or severe FI would have poorer diet quality, higher body weights and poorer psychosocial outcomes than students from households classed as having high FS or marginal FI.

\section{Methods}

The Children's Lifestyle And School performance Study (CLASS) is a population-based survey that examined nutrition, physical activity, well-being and school performance of grade 5 students (10 or 11 years old) in Nova Scotia, Canada in 2011. All Nova Scotian school students, their parents and school stakeholders were invited to participate via packages containing consent forms and a survey that were sent home with all grade 5 students. Out of 286 eligible schools, 269 (94.1\%) agreed to participate. Parental consent was obtained for 5913 students, resulting in an average response rate of $67.7 \%$ per school. Ethics approval for the study was obtained from the Health Research Ethics Boards at the University of Alberta and Dalhousie University. Permission for data collection was also granted from participating school boards.

\section{Data collection}

Trained research assistants visited schools to administer a slightly modified, Canadian version of the 147-item Harvard Youth Adolescent Food Frequency Questionnaire (YAQ) ${ }^{(23)}$ to participating students, along with an additional survey that included measures on physical and sedentary activities, mental health, self-efficacy and body image. Students were also asked about their eating behaviours at school and home, mental well-being (including mood, peer relationships and externalizing problems) and quality of life. The research assistants also measured the heights and weights of participating students. Standing height was measured to the nearest $0.1 \mathrm{~cm}$ after students had removed their shoes. Body weight was measured in light indoor clothing to the nearest $0 \cdot 1 \mathrm{~kg}$ on calibrated digital scales. The parent survey contained questions on sociodemographic factors, the home environment, their child's health and the FS status of their household. Surveys and consent forms are available from www.nsclass.ca. The seven variables included in the analysis were chosen for their fit within the conceptual framework and are listed in Fig. 1.

\section{Exposure}

FS was assessed using the six-item version of the US $\operatorname{HFSSM}^{(5,24)}$, which has been shown to have reasonably high specificity and sensitivity and minimal bias and respondent burden with respect to the eighteen-item HFSSM ${ }^{(25)}$. The FS score was derived from the number of affirmative responses to the six questions about FS. Missing values were imputed for individuals with three or fewer missing responses by following the procedure outlined in the instrument's documentation $^{(5)}$. Based on the overall score, households were then classified as having high FS (score 0), marginal FI (score 1), moderate FI (score 2-4) or severe FI (score 5-6).

\section{Outcomes}

Since some of the mechanisms in the framework act as mediators (i.e. are in the causal pathway) they cannot be used as co-exposures in a regression analysis because of the potential for over-adjustment. For the purpose of the analysis, we have therefore treated all 'mechanisms' as outcomes. The children's weight status was categorized as 


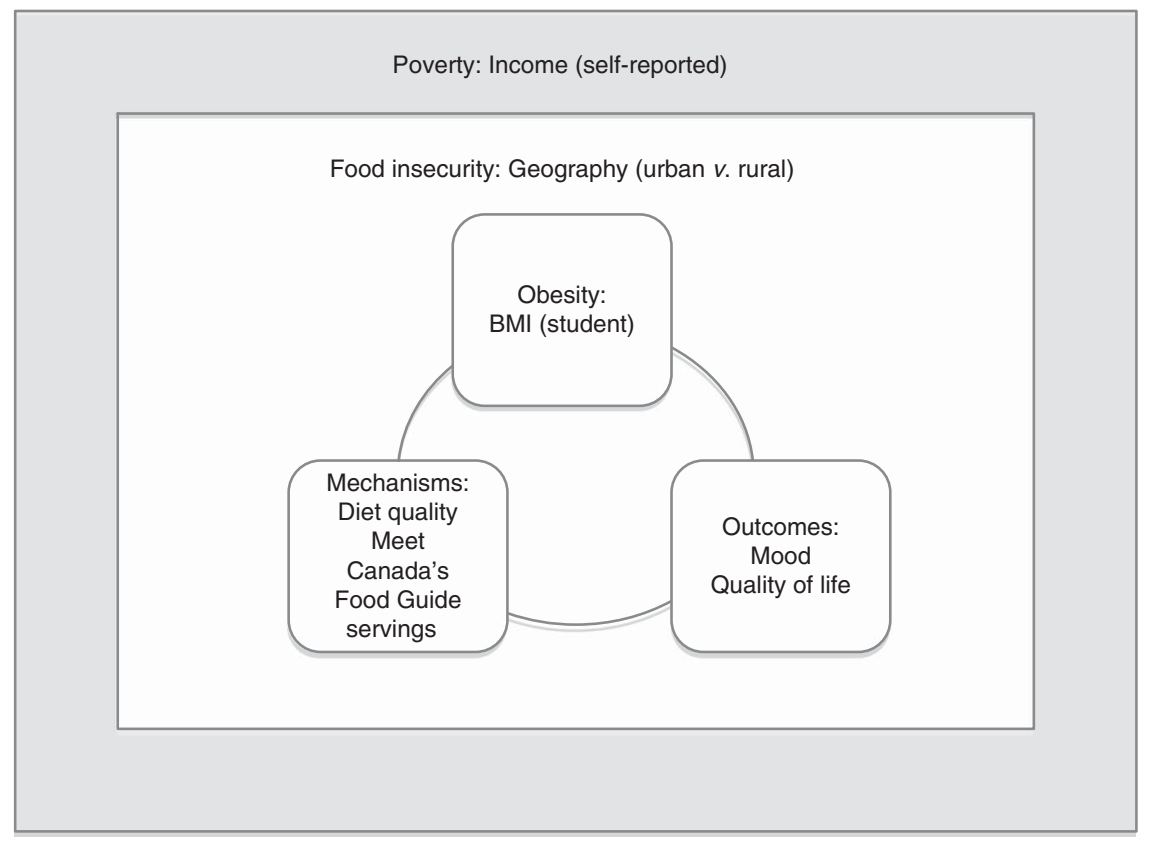

Fig. 1 Conceptual framework: poverty, food insecurity, obesity and health outcomes, adapted to include examples of variables collected through the CLASS II study

normal weight, overweight or obese, and BMI Z-scores were calculated using the LMS method based on the International Obesity Task Force reference population ${ }^{(26)}$. Other outcomes comprised quality of life, mood, peer relationships and externalizing problems. Health-related quality of life was measured with the EQ-5D-Y ${ }^{(27,28)}$. The US EQ-5D index values were used to calculate a single index score ${ }^{(29)}$. Mood, peer relationships and externalizing problems were assessed using a series of ten questions with 3-point Likert scale response options similar to the Emotional Functioning and Social Functioning items on the PedsQL ${ }^{\mathrm{TM}(30)}$. Students' diet quality and energy intake were assessed using the YAQ and nutrient information from the Canadian Nutrient File ${ }^{(31)}$. Overall diet quality was measured using the Diet Quality Index-International (DQI) score, a composite measure of diet quality ranging from 0 to $100^{(32)}$. DQI values encompass dietary variety (i.e. overall variety and variety within protein sources, to assess whether intake comes from diverse sources both across and within food groups), adequacy (i.e. the intake of dietary elements that must be supplied sufficiently to guarantee a healthy diet), moderation (i.e. intake of food and nutrients that are related to chronic diseases and that may need restriction) and balance (i.e. the overall balance of diet in terms of proportionality in energy sources and fatty acid composition). 'Empty calorie foods' is an additional measure of nutrient density within the DQI, measuring how much a person's energy supply is dependent on low-nutrient-density foods. Food group intake was assessed based on the food groups set out in Canada's Food Guide ${ }^{(33)}$ by determining whether students were meeting the recommended daily number of food group servings (yes/no) for fruits and vegetables $(\geq 6)$, milk products $(\geq 3)$, grain products $(\geq 6)$ and meat products $(\geq 1)$. Food group intakes were standardized to an energy intake of $8368 \mathrm{~kJ} / \mathrm{d}(2000 \mathrm{kcal} / \mathrm{d})$.

\section{Covariates}

Other variables used in the analysis were gender, household income (four levels: \$CAN 0-20 000, \$CAN 20 001-40 000, \$CAN 40 001-60 000, > \$CAN 60 000; $20 \%$ had either missing values or parents had indicated that they preferred not to answer), parental education attainment (three levels: secondary school or lower, college, university) and area of residence (urban, rural; based on the second character of the Forward Sortation Area in the Canadian postal code; rural postal codes contain a 0 as the second character).

\section{Statistical analysis}

All statistical analyses were weighted to represent provincial estimates of the grade 5 student population in Nova Scotia. Response rates were calculated per decile of neighbourhood household income according to postal code data from the 2011 Canadian census and converted into response weights to account for non-response bias due to lower participation rates in residential areas with lower household incomes. A series of linear and Poisson regression models with robust standard errors, to account for the clustering of students in schools, was used to examine the associations between FS status, diet and health-related outcomes. Poisson regression with robust standard errors instead of logistic regression was used to avoid overestimation of the relative risk (prevalence of the outcomes was consistently $>20 \%)^{(34)}$. Multiple regression 
models were incrementally adjusted for gender, area of residence and household education; models for dietary outcomes were further adjusted for energy intake ${ }^{(35)}$. Missing values were considered as separate covariate categories but their estimates are not presented. Normality and homoscedasticity were tested and found to be acceptable for all linear regression models. The analyses were performed using the statistical software package Stata/SE 12.

\section{Results}

A total of 5853 respondents (out of 5913 with parental consent) had complete information on FS and student outcomes; $73.5 \%$ were classified as being from households with high FS status, $8.3 \%$ from households with marginal FI status, $10 \cdot 2 \%$ from households with moderate FI status and $7 \cdot 1 \%$ from households with severe FI status, for a combined prevalence of moderate to severe FI of $17.3 \%$. For parental education attainment, $4 \%$ of the sample had either missing values or parents had indicated that they preferred not to answer. Characteristics of the sample by FS status are presented in Table 1.

Tables 2 and 3 present the results of the linear, logistic and Poisson regression analyses of dietary, weight and psychosocial outcomes across levels of FS status. For dietary outcomes, compared with students living in households with high FS status, students living in households with moderate or severe FI reported a higher energy intake in the univariate and adjusted models, with this being statistically significant for students living in households reporting severe FI. Moderate and severe FI were also associated with lower overall DQI, DQI variety, DQI adequacy, DQI moderation and a lower quality of life.

In the models adjusted for gender and area of residence, students from households experiencing marginal, moderate and severe FI had statistically significantly higher BMI and were less likely to meet the recommendations in Canada's Food Guide for fruit and vegetables and milk products. Students in households with moderate or severe FI were significantly more likely to report relationship, mood or externalizing problems. For all models, further adjustment for household income reduced the magnitude

Table 1 Characteristics of grade 5 students (aged 10-11 years) in Nova Scotia, Canada by food security status, Children's Lifestyle And School performance Study (CLASS), 2011. Results are presented as proportions or as means and standard deviations as applicable

\begin{tabular}{|c|c|c|c|c|c|c|c|c|}
\hline & \multicolumn{8}{|c|}{ FS status } \\
\hline & \multicolumn{2}{|c|}{ High FS (n 4356) } & \multicolumn{2}{|c|}{ Marginal FI (n 472) } & \multicolumn{2}{|c|}{ Moderate FI ( $n$ 570) } & \multicolumn{2}{|c|}{ Severe FI (n 401) } \\
\hline & Mean & SD & Mean & SD & Mean & SD & Mean & SD \\
\hline Male gender (\%) & \multicolumn{2}{|c|}{49} & \multicolumn{2}{|c|}{43} & \multicolumn{2}{|c|}{46} & \multicolumn{2}{|c|}{44} \\
\hline Rural residence (\%) & \multicolumn{2}{|c|}{34} & \multicolumn{2}{|c|}{38} & \multicolumn{2}{|c|}{40} & \multicolumn{2}{|c|}{39} \\
\hline \$CAN 0-20000 & \multicolumn{2}{|c|}{15} & \multicolumn{2}{|c|}{41} & \multicolumn{2}{|c|}{55} & \multicolumn{2}{|c|}{75} \\
\hline$\$ C A N 20001-40000$ & \multicolumn{2}{|c|}{16} & \multicolumn{2}{|c|}{26} & \multicolumn{2}{|c|}{25} & \multicolumn{2}{|c|}{18} \\
\hline \$CAN $40001-60000$ & \multicolumn{2}{|c|}{37} & \multicolumn{2}{|c|}{28} & \multicolumn{2}{|c|}{19} & \multicolumn{2}{|c|}{6} \\
\hline$>$ \$CAN 60000 & \multicolumn{2}{|c|}{33} & \multicolumn{2}{|c|}{6} & \multicolumn{2}{|c|}{2} & & \\
\hline Household education (\%) & & & & & & & & \\
\hline Secondary school or less & & & & & & & & \\
\hline College & & & & & & & & \\
\hline University & & & & & & & & \\
\hline BMI Z-score & 0.73 & $1 \cdot 12$ & 0.92 & 1.09 & 0.88 & $1 \cdot 18$ & 0.90 & $1 \cdot 17$ \\
\hline Weight status (\%) & & & & & & & & \\
\hline Underweight & & & & & & & & \\
\hline Normal weight & & & & & & & & \\
\hline Overweight & & & & & & & & \\
\hline Obese & & & & & & & & \\
\hline EQ-5D index score & 0.89 & 0.14 & 0.87 & 0.14 & 0.86 & 0.15 & 0.86 & 0.16 \\
\hline Externalizing problems (\%) & & & & & & & & \\
\hline Relationship problems (\%) & & & & & & & & \\
\hline Mood problems (\%) & & & & & & & & \\
\hline Energy intake $(\mathrm{kJ} / \mathrm{d})$ & 7780 & 3274 & 8049 & 3501 & 8390 & 3587 & 8437 & 3621 \\
\hline DQI & $62 \cdot 6$ & $9 \cdot 8$ & $62 \cdot 1$ & $9 \cdot 3$ & $61 \cdot 6$ & $10 \cdot 1$ & $61 \cdot 7$ & $10 \cdot 2$ \\
\hline DQI variety & $15 \cdot 3$ & $4 \cdot 0$ & $15 \cdot 3$ & $4 \cdot 0$ & $15 \cdot 1$ & $4 \cdot 1$ & $15 \cdot 2$ & $4 \cdot 0$ \\
\hline DQI adequacy & $29 \cdot 3$ & $5 \cdot 8$ & $29 \cdot 3$ & $5 \cdot 7$ & $29 \cdot 3$ & $6 \cdot 1$ & 29.5 & $6 \cdot 2$ \\
\hline DQI moderation & $16 \cdot 7$ & $5 \cdot 1$ & $16 \cdot 1$ & $5 \cdot 1$ & $15 \cdot 7$ & 4.9 & $15 \cdot 4$ & 4.9 \\
\hline DQI balance & $1 \cdot 3$ & $1 \cdot 8$ & 1.4 & $1 \cdot 8$ & 1.4 & $1 \cdot 8$ & $1 \cdot 7$ & 1.9 \\
\hline DQI empty calories (\%) & & & & & & & & \\
\hline Meets fruit \& vegetable intake recommendations (\%) & & & & & & & & \\
\hline Meets milk product intake recommendations (\%) & & & & & & & & \\
\hline Meets grain product intake recommendations (\%) & & & & & & & & \\
\hline Meets meat product intake recommendations (\%) & & & & & & & & \\
\hline
\end{tabular}

FS, food security; FI, food insecurity; DQI, Diet Quality Index. 
Table 2 Linear regression: relationship between food security and dietary and psychosocial outcomes among grade 5 students (aged 10-11 years) in Nova Scotia, Canada, Children's Lifestyle And School performance Study (CLASS), 2011

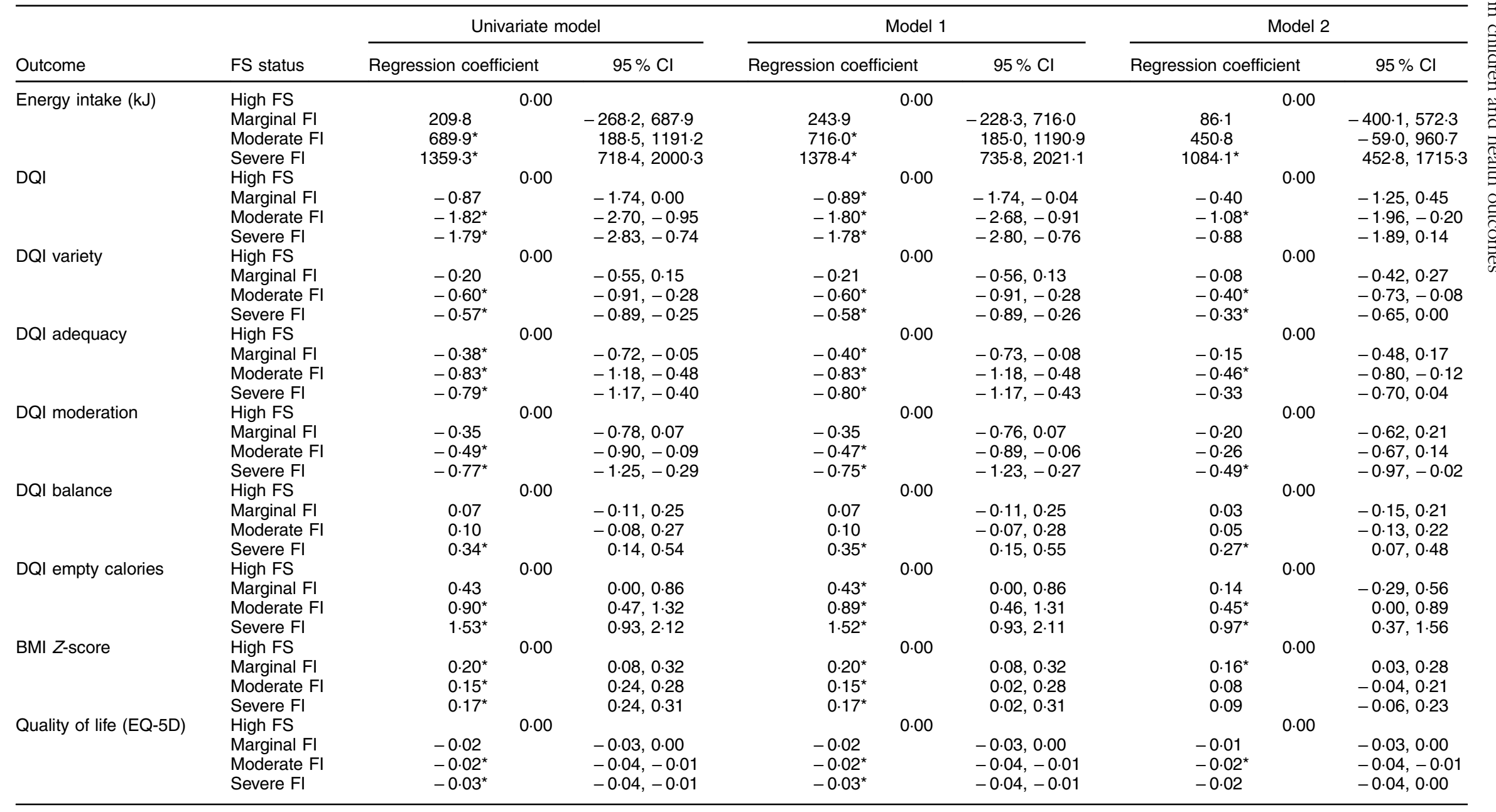

FS, food security; FI, food insecurity; DQI, Diet Quality Index.

Model 1, adjusted for gender and area of residence; Model 2, Model 1 + education; DQI-I models were also adjusted for energy intake.

${ }^{*} P<0.05$. 
Table 3 Poisson regression: relationship between food security and dietary and psychosocial outcomes among grade 5 students (aged 10-11 years) in Nova Scotia, Canada, Children's Lifestyle And School performance Study (CLASS), 2011

\begin{tabular}{|c|c|c|c|c|c|c|c|}
\hline \multirow[b]{2}{*}{ Outcome } & \multirow[b]{2}{*}{ FS status } & \multicolumn{2}{|c|}{ Univariate model } & \multicolumn{2}{|c|}{ Model 1} & \multicolumn{2}{|c|}{ Model 2} \\
\hline & & PR & $95 \% \mathrm{Cl}$ & PR & $95 \% \mathrm{Cl}$ & PR & $95 \% \mathrm{Cl}$ \\
\hline \multirow{4}{*}{$\begin{array}{l}\text { Meets fruit and vegetable intake } \\
\text { recommendations }\end{array}$} & High FS & \multicolumn{2}{|c|}{1.00} & \multicolumn{2}{|c|}{1.00} & \multicolumn{2}{|c|}{1.00} \\
\hline & Marginal FI & $0.83^{\star}$ & $0.70,0.99$ & $0.82^{\star}$ & $0.69,0.97$ & 0.87 & $0.74,1.03$ \\
\hline & Moderate FI & $0.79^{*}$ & $0.67,0.93$ & $0.79^{*}$ & $0.67,0.93$ & 0.86 & $0.73,1.02$ \\
\hline & Severe FI & $0.73^{\star}$ & $0.59,0.90$ & $0.73^{*}$ & $0.59,0.89$ & $0.81^{*}$ & $0.66,1.00$ \\
\hline \multirow[t]{4}{*}{ Meets milk intake recommendations } & High FS & \multicolumn{2}{|c|}{1.00} & \multicolumn{2}{|c|}{1.00} & \multicolumn{2}{|c|}{1.00} \\
\hline & Marginal FI & $0.86^{*}$ & $0.77,0.96$ & $0.86^{*}$ & $0.78,0.96$ & $0.89^{*}$ & $0.80,0.98$ \\
\hline & Moderate FI & $0.88^{*}$ & $0.80,0.97$ & $0.89^{\star}$ & $0.80,0.98$ & 0.92 & $0.83,1.02$ \\
\hline & Severe FI & $0.82^{*}$ & $0.83,0.94$ & $0.83^{*}$ & $0.73,0.94$ & $0.87^{\star}$ & $0.76,0.99$ \\
\hline \multirow[t]{4}{*}{ Meets grain intake recommendations } & High FS & \multicolumn{2}{|c|}{1.00} & \multicolumn{2}{|c|}{1.00} & \multicolumn{2}{|c|}{1.00} \\
\hline & Marginal FI & 1.03 & $0.84,1.25$ & 1.04 & $0.86,1.27$ & 1.07 & $0.88,1.30$ \\
\hline & Moderate FI & $1 \cdot 27^{\star}$ & $1.06,1.53$ & $1 \cdot 28^{*}$ & $1.06,1.54$ & $1 \cdot 33^{*}$ & $1.10,1.62$ \\
\hline & Severe FI & $1 \cdot 45^{\star}$ & $1.22,1.73$ & $1.47^{*}$ & $1.23,1.75$ & $1.55^{*}$ & $1.29,1.86$ \\
\hline \multirow{4}{*}{ Meets meat intake recommendations } & High FS & \multicolumn{2}{|c|}{1.00} & \multicolumn{2}{|c|}{1.00} & \multicolumn{2}{|c|}{1.00} \\
\hline & Marginal FI & 1.00 & $0.96,1.04$ & 1.00 & $0.96,1.04$ & 1.00 & $0.97,1.05$ \\
\hline & Moderate FI & 0.97 & $0.93,1.01$ & 0.97 & $0.93,1.01$ & 0.98 & $0.93,1.02$ \\
\hline & Severe FI & 1.00 & $0.96,1.05$ & 1.00 & $0.96,1.05$ & 1.01 & $0.97,1.06$ \\
\hline \multirow[t]{4}{*}{ Relationship problems } & High FS & \multicolumn{2}{|c|}{1.00} & \multicolumn{2}{|c|}{1.00} & \multicolumn{2}{|c|}{1.00} \\
\hline & Marginal FI & $1 \cdot 11$ & $0.95,1.30$ & $1 \cdot 11$ & $0.94,1.30$ & 1.06 & $0.90,1.24$ \\
\hline & Moderate FI & $1 \cdot 29^{*}$ & $1 \cdot 15,1 \cdot 44$ & $1 \cdot 28^{*}$ & $1 \cdot 13,1 \cdot 44$ & $1 \cdot 19^{\star}$ & $1.05,1.34$ \\
\hline & Severe FI & $1 \cdot 28^{*}$ & $1.12,1.45$ & $1 \cdot 29^{*}$ & $1 \cdot 12,1.48$ & $1 \cdot 18^{*}$ & $1.03,1.36$ \\
\hline \multirow[t]{4}{*}{ Mood problems } & High FS & \multicolumn{2}{|c|}{1.00} & \multicolumn{2}{|c|}{1.00} & \multicolumn{2}{|c|}{1.00} \\
\hline & Marginal FI & $1 \cdot 28^{*}$ & $1 \cdot 13,1 \cdot 44$ & $1 \cdot 27^{*}$ & $1 \cdot 12,1.43$ & $1 \cdot 21^{*}$ & $1.07,1.37$ \\
\hline & Moderate FI & $1 \cdot 36^{\star}$ & $1.23,1.50$ & $1.34^{\star}$ & $1.21,1.49$ & $1.25^{\star}$ & $1.13,1.40$ \\
\hline & Severe FI & $1 \cdot 33^{*}$ & $1 \cdot 19,1 \cdot 48$ & $1 \cdot 32^{\star}$ & $1 \cdot 18,1.49$ & $1.22^{*}$ & $1.09,1.37$ \\
\hline \multirow[t]{4}{*}{ Externalizing problems } & High FS & & & & & & \\
\hline & Marginal FI & $1 \cdot 12$ & $0.96,1.31$ & $1 \cdot 13$ & $0.97,1.33$ & 1.08 & $0.92,1.27$ \\
\hline & Moderate FI & $1 \cdot 30^{*}$ & $1 \cdot 16,1 \cdot 46$ & $1 \cdot 30^{*}$ & $1 \cdot 15,1.46$ & $1 \cdot 20^{*}$ & $1.07,1.35$ \\
\hline & Severe FI & $1.41^{*}$ & $1 \cdot 24,1 \cdot 61$ & $1.46^{*}$ & $1 \cdot 28,1 \cdot 67$ & $1 \cdot 33^{\star}$ & $1.16,1.52$ \\
\hline
\end{tabular}

FS, food security; PR, prevalence ratio; FI, food insecurity.

Model 1, adjusted for gender and area of residence; Model 2, Model 1 + education.

${ }^{*} P 0.05$.

and statistical significance of all associations substantially (data not shown).

\section{Discussion}

The provincial focus and large sample size of the current study provided an accurate estimate of the prevalence of FI in Nova Scotian households with grade 5 children; a total of $17.3 \%$ of families with grade 5 children reported experiencing moderate to severe FI. The most recent data on FI in families with children, taken from the Canadian Community Health Survey, estimated a prevalence of $23 \%$ among Nova Scotian families in 2011, which is higher than the figure reported here and includes children of all ages $^{(7)}$. The current study provides an important estimate of FI prevalence in households with grade 5 students, as well as further, substantive evidence to suggest that difficulties in accessing sufficient, safe and nutritious food is an issue for a concerningly high number of families with children in Nova Scotia.

Consistent with our hypothesis, students living in households experiencing moderate or severe FI had poorer diet quality, higher BMI and poorer psychosocial outcomes than students classed as having high FS. In terms of meeting recommendations in Canada's Food Guide, moderate and severe FI was negatively associated with meeting intake recommendations for vegetables and fruits and milk products, while the reverse was seen for meeting grain product recommendations. No difference was observed between different levels of household FS for meeting meat/alternatives intake recommendations. It is possible that the meat and alternatives food group is prioritized over other food groups, as has been suggested in a previous qualitative study in Quebec ${ }^{(36)}$. This seems plausible, given that the DQI variety score was lower in students from households experiencing moderate or severe FI. Alternatively, it could be that the measure used (FFQ) was not sufficiently sensitive to detect differences in this food group. Taken with the findings that students experiencing moderate or severe FI also reported higher energy intakes, it could be that moderate to severe FI in these students impacted diet quality, possibly through greater reliance on energy-dense, nutrient-poor foods, or through less variety within protein sources. Our findings also concur with earlier findings that adults and adolescents in households with low levels of income or those on social assistance are less likely to purchase milk products ${ }^{(15)}$, fruits and vegetables or to consume the recommended daily servings of fruits and vegetables ${ }^{(13,14)}$. Although it is 
not possible to confirm that poor diet quality acts as a mechanism for obesity as outlined in the conceptual framework proposed by Finney Rutten et $a l^{(22)}$, these findings suggest that poor diet quality is indeed an important factor influencing the FI and obesity relationship.

Of note, associations between FI and the study outcomes were reduced in magnitude and for the most part no longer statistically significant after adjustment for household income. This finding is likely due to an overadjustment of the model since the items in the HFSSM module assess situations that are closely linked to insufficient money to afford food. We therefore feel that the results from Model 2 better reflect the relationship between FI and the study outcomes.

That students experiencing FI also experienced psychosocial stress is not surprising given previous research, but what is concerning is that some of these problems were significantly more prevalent even in students living in households with marginal FI. FI has been linked to stress and impaired academic performance in US children ${ }^{(37)}$ and qualitative data from families living in the Canadian province of Quebec highlighted the stressors felt by parents in not being able to provide adequate food for their children ${ }^{(36)}$, including disruption to family meal-times or having to stop the child from continuing to eat, even though he/she was still hungry, because there was not enough food ${ }^{(38)}$. As previously outlined, qualitative studies also highlight that adults, and in some cases adolescents, in food insecure households prioritize food provision to children in the household over their own needs ${ }^{(11,36,39)}$.

The negative impact of moderate to severe FI was also seen in significantly lower health-related quality of life outcomes. We found only one other study that explored this relationship ${ }^{(40)}$. This US-based study used the same FS survey and found that FI was related to lower physical, psychosocial and total quality of life scores among 3- to 17 -year-old children and youth ${ }^{(40)}$. The physical and total quality of life scores were lower among 3- to 8-year-old children, and the psychosocial and total quality of life scores were lower among FI youth aged 12 to 17 years, while 9- to 11-year-old children did not have significant quality of life impacted by FI. An earlier Canadian study of grade 5 students found that children reporting better diet quality, higher physical activity and normal body weight were statistically significantly more likely to report better quality of life than children with low diet quality, physical inactivity and overweight or obesity ${ }^{(41)}$. Both of these previous studies indicated that FS status and quality of life were at least partly related to these three factors $^{(40,41)}$, which we have further elucidated in the present study.

The current study has several strengths, including the use of a large, population-based sample of grade 5 students with a high response rate and the reduction of nonresponse bias through the use of weighting. Despite the use of weighting, selection bias due to non-response cannot be excluded. As response rates were lower in socio-economically disadvantaged areas, our findings may have underestimated the prevalence of FI. The students' BMI is based on measured (as opposed to self-reported) height and weight, and the instruments used to assess FS and diet are validated and widely used. However, self-reported response is inherently subjective and may be prone to error. A further limitation is the cross-sectional nature of the data that does not allow exploration of cause and effect in the relationships identified. Future analyses in this sample will seek to explore the interrelationships between determinants and outcomes of FS in a structural equation model.

The findings of the present research have important implications for policy makers both provincially and elsewhere, particularly given that FI remains a significant global challenge ${ }^{(42)}$. Prior research in Nova Scotia provided important insight on poor nutritional habits and high prevalence of overweight and obesity among grade 5 children ${ }^{(43,44)}$; those research findings subsequently led to the development of various policy investments to create healthier school environments ${ }^{(45,46)}$. The CLASS research was conducted to investigate the impact of these strategies and explore issues of relevance to Nova Scotian stakeholders, such as FI and its determinants. The current study builds on this earlier work and provides important evidence for policy makers on the prevalence of FI among Nova Scotian families and its relationship with childhood nutrition, psychosocial factors and overweight and obesity. Although limited to Nova Scotia, our findings have implications elsewhere, particularly when considered in the context of an increase in food prices that is being observed globally ${ }^{(42)}$.

It is clear that household income is an important predictor of FS status, since adjusting for this substantially reduced the magnitude and statistical significance of all associations. The relationships established in the current study therefore provide a compelling case for policy makers to consider actions that address the social-structural conditions of FI, such as low income and poverty, as well the increasing costs of healthy foods ${ }^{(14,47)}$. Based on the study results, such broad actions could not only help to address the issue of FI but also improve nutrition behaviours and psychosocial factors and reduce rates of obesity among children.

\section{Conclusions}

The present study adds to a growing body of evidence that FI does impact diet quality, promoting consumption of energy-dense, nutrient-poor foods at the expense of foods of higher nutritional value, which in turn may lead to overweight and obesity and other diet-related health problems. It highlights the importance of upstream approaches that address the issues of poverty and FI in 
communities and among households, particularly those with children ${ }^{(48)}$, and the important but often neglected aspect of mental health and well-being. Advocacy and awareness of the implications of FI requires ground-swell support from the community. Policy interventions, such as providing a liveable income, as well as best start practices for early childhood development, require political will and the adoption of a rights-based approach that recognizes the right to food as synonymous with the right to health ${ }^{(49)}$. Finally, creating supportive school environments that encourage children and families to be sensitive to the health and the psychosocial impacts of FI highlights the role of schools in supporting students who may be experiencing FI, both through comprehensive approaches to health that recognize the importance of mental health and through the provision of universal feeding programmes (e.g. breakfast and lunch programmes) that mitigate stigma and support FS for all.

\section{Acknowledgements}

This manuscript is dedicated to the memory of Hannah Carmichael. Acknowledgements: The authors would like to thank Dr Mark Nord, US Department of Agriculture, for his support with the scoring of the FS questionnaire, and Connie Lu, University of Alberta, for data management. Financial support: This work was supported by the Canadian Institutes for Health Research (CIHR; operating grant number CIHR FRN 93680 to P.J.V. and S.F.L.K.), the Vanier Canada Graduate Scholarships Program (to J.-L.D.M.), a Canada Research Chair in Population Health (to P.J.V.) and Health Services Research (to S.F.L.K.). Salary support is also acknowledged from an Alberta Innovates Health Scholarship (to P.J.V.). Funders had no role in the design, analysis or writing of this article. Conflict of interest: None. Authorship: S.F.L.K., P.J.V., P.L.W. and M.R. acquired funding for the CLASS II project and designed and conducted the CLASS II study. J.-L.D.M. coordinated data collection. S.F.L.K., S.K. and J.-L.D.M. developed the analysis plan for this manuscript. S.K. conducted the analysis. S.F.L.K. drafted the manuscript with input from all authors. All authors provided critical review of the manuscript. P.L.W. provided expertise on measuring food security, A.O. provided expertise on measuring quality of life. Ethics of human subject participation: Ethics approval for the study was obtained from the Health Research Ethics Boards at the University of Alberta and Dalhousie University. Permission for data collection was also granted from participating school boards.

\section{References}

1. World Food Conference General Assembly (1974) Universal Declaration on the Eradication of Hunger and malnutrition. http://www.ohchr.org/EN/ProfessionalInterest/ Pages/EradicationOfHungerAndMalnutrition.aspx (accessed July 2014).
2. Food and Agriculture Organization (1996) Rome Declaration on World Food Security and World Food Summit Plan of Action. http://www.fao.org/docrep/003/w3613e/w3613e00. htm (accessed July 2014).

3. Tarasuk V (2001) Discussion paper on household and individual food insecurity. http://www.hc-sc.gc.ca/fn-an/ alt_formats/hpfb-dgpsa/pdf/nutrition/food_sec_entire-sec_ aliments_entier-eng.pdf (accessed July 2014).

4. Health Canada (2012) Household Food Insecurity In Select Provinces and the Territories in 2009-2010. http:// www.hc-sc.gc.ca/fn-an/surveill/nutrition/commun/insecurit/ prov_ter-eng.php (accessed July 2014).

5. Bickel G, Nord M, Price C et al. (2000) Guide to Measuring Housebold Food Security, Revised 2000. Alexandria, VA: USDA/FNS; available at http://www.fns.usda.gov/fsec/FILES/ FSGuide.pdf

6. Statistics Canada (2010) Household Food Insecurity 2007-2008: Canadian Community Health Survey. http://www.statcan. gc.ca/pub/82-625-x/2010001/article/11162-eng.htm （accessed June 2013).

7. Tarasuk V, Mitchell A \& Dachner N (2013) Research to identify policy options to reduce food insecurity (PROOF). Household food insecurity in Canada 2011. http://nutritional sciences.lamp.utoronto.ca/resources/proof-annual-reports/ annual-report-2012/ (accessed July 2014).

8. Vozoris NT \& Tarasuk VS (2003) Household food insufficiency is associated with poorer health. J Nutr 133, 120-126.

9. Health Canada (2007) Canadian Community Health Survey, cycle 2.2, nutrition (2004) - income-related household food security in Canada. http://www.hc-sc.gc.ca/fn-an/surveill/ nutrition/commun/income_food_sec-sec_alim-eng.php (accessed July 2014).

10. Willows ND, Veugelers P, Raine K et al. (2009) Prevalence and sociodemographic risk factors related to household food security in Aboriginal peoples in Canada. Public Health Nutr 12, 1150-1156.

11. Hamelin AM, Habicht JP \& Beaudry M (1999) Food insecurity: consequences for the household and broader social implications. J Nutr 129, 2S Suppl, 525S-528S.

12. Power EM (2005) Determinants of healthy eating among low-income Canadians. Can J Public Health 96, Suppl. 3, S37-S42.

13. Kirkpatrick SI \& Tarasuk V (2008) Food insecurity is associated with nutrient inadequacies among Canadian adults and adolescents. J Nutr 138, 604-612.

14. Ricciuto LE \& Tarasuk VS (2007) An examination of income-related disparities in the nutritional quality of food selections among Canadian households from 1986-2001. Soc Sci Med 64, 186-198.

15. Williams PL, McIntyre L \& Glanville T (2010) Milk insecurity: accounts of a food insecurity phenomenon in Canada and its relation to public policy. J Hunger Environ Nutr 5, 142-157.

16. Dietz WH (1995) Does hunger cause obesity? Pediatrics 95 , 766-767.

17. Dinour LM, Bergen D \& Yeh MC (2007) The food insecurity-obesity paradox: a review of the literature and the role food stamps may play. J Am Diet Assoc 107, 1952-1961.

18. Tanumihardjo SA, Anderson C, Kaufer-Horwitz M et al. (2007) Poverty, obesity, and malnutrition: an international perspective recognizing the paradox. J Am Diet Assoc 107, 1966-1972.

19. Townsend MS, Peerson J, Love B et al. (2001) Food insecurity is positively related to overweight in women. J Nutr 131, 1738-1745.

20. Martin MA \& Lippert AM (2012) Feeding her children, but risking her health: the intersection of gender, household food insecurity and obesity. Soc Sci Med 74, 1754-1764.

21. Franklin B, Jones A, Love D et al. (2012) Exploring mediators of food insecurity and obesity: a review of recent literature. J Community Health 37, 253-264. 
22. Finney Rutten LJ, Yaroch AL, Colon-Ramos U et al. (2010) Poverty, food insecurity and obesity: a conceptual framework for research, practice and policy. I Hunger Environ Nutr 5, 403-415.

23. Rockett HR, Wolf AM \& Colditz GA (1995) Development and reproducibility of a food frequency questionnaire to assess diets of older children and adolescents. I Am Diet Assoc 95, 336-340.

24. Nord M, Andrews M \& Carlson S (2009) Housebold Food Security in the United States, 2008. Economic Research Report no. ERR-66. Washington, DC: USDA/ERS.

25. Blumberg SJ, Bialostosky K, Hamilton WL et al. (1999) The effectiveness of a short form of the Household Food Security Scale. Am J Public Health 89, 1231-1234.

26. Cole TJ \& Lobstein T (2012) Extended international (IOTF) body mass index cut-offs for thinness, overweight and obesity. Pediatr Obes 7, 284-294.

27. EuroQol Group (1990) EuroQol EQ-5D: a new facility for the measurement of health-related quality of life. Health Policy 19, 199-208.

28. Wille N, Badia X, Bonsel G et al. (2010) Development of the EQ-5D-Y: a child-friendly version of the EQ-5D. Qual Life Res 19, 875-886.

29. Shaw JW, Johnson JA \& Coons SJ (2005) US evaluation of the EQ-5D health states: development and testing of the D1 valuation model. Med Care 43, 203-220.

30. Varni JW (2013) The PedsQL ${ }^{\mathrm{TM}}$ : Measurement Model for the Pediatric Quality of Life Inventory. http://www.pedsql.org/ (accessed May 2013).

31. Health Canada (2007) Canadian Nutrient File. http:// www.hc-sc.gc.ca/fn-an/nutrition/fiche-nutri-data/cnf_aboutusaproposdenous_fcen-eng.php (accessed November 2012).

32. Kim S, Haines PS, Siega-Riz AM et al. (2003) The Diet Quality Index-International (DQI-I) provides an effective tool for cross-national comparison of diet quality as illustrated by China and the United States. I Nutr 133 , 3476-3484.

33. Health Canada (2008) Eating Well with Canada's Food Guide, 2007. http://www.hc-sc.gc.ca/fn-an/food-guide-aliment/ index_e.html (accessed December 2008).

34. Barros AJ \& Hirakata VN (2003) Alternatives for logistic regression in cross-sectional studies: an empirical comparison of models that directly estimate the prevalence ratio. BMC Res Methodol 3, 21.

35. Willett W (1998) Nutritional Epidemiology. New York: Oxford University Press.

36. Hamelin AM, Beaudry M \& Habicht JP (2002) Characterization of household food insecurity in Quebec: food and feelings. Soc Sci Med 54, 119-132.
37. Jyoti DF, Frongillo EA \& Jones SJ (2005) Food insecurity affects school children's academic performance, weight gain, and social skills. I Nutr 135, 2831-2839.

38. Alaimo K, Olson CM \& Frongillo EA (2002) Family food insufficiency, but not low income is positively associated with dysthymia and suicide symptoms in adolescents. $J$ Nutr 132, 719-725.

39. Williams PL, MacAulay RB, Anderson BJ et al. (2012) 'I would have never thought that I would be in such a predicament': voices from women experiencing food insecurity in Nova Scotia, Canada. J Hunger Environ Nutr 7, 253-270.

40. Casey P, Szeto K, Robbin J et al. (2005) Child health-related quality of life and household food security. Arch Pediatr Adolesc Med 159, 51-56.

41. Wu XY, Ohinmaa A \& Veugelers PJ (2012) Diet quality, physical activity, body weight and health-related quality of life among grade 5 students in Canada. Public Health Nutr 15, 75-81.

42. Food and Agriculture Organization of the United Nations (2012) The State of Food Insecurity in the World 2012. Rome: FAO; available at http://www.fao.org/docrep/016/ i3027e/i3027e.pdf

43. Veugelers PJ \& Fitzgerald AL (2005) Prevalence of and risk factors for childhood overweight and obesity. CMAJ 173, 607-613

44. Veugelers PJ, Fitzgerald AL \& Johnston E (2005) Dietary intake and risk factors for poor diet quality among children in Nova Scotia. Can I Public Health 96, 212-216.

45. McIsaac JL, Sim SM, Penney TL et al. (2012) School health promotion policy in Nova Scotia: a case study. PHEnex $J$ 4(2). http://ojs.acadiau.ca/index.php/phenex/article/view/ 1460 (accessed July 2014).

46. Fung C, McIsaac JL, Kuhle S et al. (2013) The impact of a population-level school food and nutrition policy on dietary intake and body weights of Canadian children. Prev Med 57, 934-940.

47. Nova Scotia Participatory Food Costing Project (2013) Can Nova Scotians Afford to Eat Healthy? Report on 2012 Participatory Food Costing. http://foodarc.ca/wp-content/ uploads/2013/08/2012-Food-Costing-Report_Final_website. pdf (accessed July 2014).

48. Carroll-Scott A, Gilstad-Hayden K, Rosenthal L et al. (2013) Disentangling neighborhood contextual associations with child body mass index, diet, and physical activity: the role of built, socioeconomic, and social environments. Soc Sci Med 95, 106-114.

49. Dowler EA \& O'Connor D (2012) Rights-based approaches to addressing food poverty and food insecurity in Ireland and UK. Soc Sci Med 74, 44-51. 